\title{
Alzheimer's disease genetic mutations: mini review
}

\begin{abstract}
Alzheimer's disease is poorly understood and continuously evolving with research. There have been many genetic mutations linked to Alzheimer's disease. Amyloid precursor protein (APP), presenilin 1 (PSEN1), and presenilin 2 (PSEN2) have been identified as causative gene mutations with early onset of Alzheimer's disease. Another genetic factor of $\mathrm{AD}$ is apolipoprotein $\mathrm{E}$ (APOE) which has been associated with the more common form of $\mathrm{AD}$-late onset Alzheimer's disease. Understanding the genetic involvement of Alzheimer's disease may lead us to early detection, prevention, and ultimately definitive treatment or even a cure. In conclusion, the genetic factors of Alzheimer's disease.
\end{abstract}

Keywords: alzheimer's disease, amyloid precursor protein, presenilin 1 (psen1), presenilin 2 (psen2), apolipoprotein e (APOE), genetics

\author{
Volume 3 Issue I - 2016
}

\author{
Nye MaryKatherine,' Wagilla Osman,' \\ Newton Suzette,' Zuba Christina,' Brown \\ Tony ${ }^{2}$ \\ 'Global Neuroscience Initiative Foundation, USA \\ ${ }^{2}$ Columbia University, USA
}

Correspondence: Tony Brown, Columbia University, New York, USA, Tel 2679736I56, Email tbrown@post.harvard.edu

Received: December 23, 2015 | Published: January 27, 2016
Abbreviations: AD, alzheimer's disease; APP, amyloid precursor protein; PSEN1, presenilin 1; PSEN2, presenilin 2; APOE, apolipoprotein E; PICALM, phosphatidylinositol binding clathrin assembly protein; TREM2, triggering receptor expressed on myeloid cells 2 ; LBD, lewy body disease

\section{Introduction}

Alzheimer's disease is one of the least understood brain disorders. The genetic association of Alzheimer's disease (AD) is an area that attracts the interest of neuroscientists since it is similar to other neurological illnesses but quite a complex disorder. It is considered to be a genetically dichotomous disease exhibiting two currently documented forms known as early onset familial cases that usually characterized by Mendelian inheritance and late onset after age 65 , with no consistent mode of transmission. ${ }^{1}$ Alzheimer's disease is an autosomal dominant inheritance disorder. Most families with AD typically develop symptoms between the ages of 30 to 60years old. The greatest risk factor of Alzheimer's is age. Genetic predisposition for $\mathrm{AD}$ has been in more current times and a topic of interest. The genetic mutations of Alzheimer's are still being researched and poorly understood; however, early onset Alzheimer's genetic mutations are better understood.

\section{Discussion}

Amyloid precursor protein (APP), presenilin 1 (PSEN1), and presenilin 2 (PSEN2) have been identified as causative gene mutations with early onset of Alzheimer's disease..$^{2-4}$ APP gene is located on chromosome 21q and encodes the protein product. ${ }^{5}$ The APP (amyloid precursor protein) forms into the $A \beta$ ( $\beta$-amyloid) peptide that is neurotoxic in the brain. It is the accumulation of $A \beta$ in the brain along with cleaved forms of microtubule protein tau that lead to Alzheimer's dementia. The exact mechanism to why $\mathrm{A} \beta$ accumulates in the brains of elderly is not completely understood at this time. ${ }^{6}$ More than 30 mutations of the APP gene have been identified in the association with $\mathrm{AD}$ and account for approximately $10-15 \%$ of early onset familial Alzheimer's Disease. The function of APP is still being researched but it is believed to be an important player in the synaptic transmission. Roughly half of early-onset AD pedigrees have been associated with mutations in PSEN1 and PSEN2, primarily in PS1. Presenilin 1 gene is located on chromosome $14 \mathrm{q}$ and encodes the protein product. ${ }^{7}$ More than 150 PSEN1 mutations have been identified with early onset of $\mathrm{AD}$. Accounting for $50 \%$ of early onset familial $\mathrm{AD}$, mutations fully penetrate and associate with earlier median age of onset compared to APP and PSEN2. PSEN1 has several different functions including regulation of intracellular calcium signaling, cell cycle and cell death, trafficking of membrane proteins, regulation of Beta-catenin stability, and Notch signalling. Small deletions and insertions have been described and most mutations of PSEN1 are missense mutations. ${ }^{8}$

Presenilin 2 gene is located on chromosome $1 \mathrm{q}$ and encodes the protein product PSEN2. ${ }^{9}$ PSEN2 is the rarest form of early onset AD and has fewer than 20 PSEN2 mutations. The age of onset is later than PSEN1 and APP Alzheimer's. PSEN2 is also thought to be estimated $95 \%$ penetrant whereas APP and PSEN1 are thought to be fully penetrant. ${ }^{10}$ PSEN2 are thought to act in the part of enhancing the apoptotic activity which leads to neurodegeneration. ${ }^{11}$ PSEN2 mutations alter the cleavage activity of PSEN2 mutations alter the cleavage activity of $y$-secretase and increase the ratio of $A \beta 42$ to A $\beta 40$ which is similar to PSEN1 mutations. Another genetic factor of $\mathrm{AD}$ is apolipoprotein $\mathrm{E}$ (APOE) which has been associated with the more common form of AD -late onset Alzheimer's disease. ${ }^{12}$ The APOE gene or apolipoprotein E has 3 common alleles in humans, which are numbered 2,3, and 4 . The APOE-4 allele is present in $20 \%$ to $30 \%$ of the general population but in $45 \%$ to $60 \%$ of patients with AD; APOE- $4 / 4$ homozygotes constitute approximately $2 \%$ to $3 \%$ of the general population but $12 \%$ to $15 \%$ of patients with AD. Many APOE-4/4 homozygotes remain cognitively healthy at advanced ages. ${ }^{12}$ Apolipoprotein E-4 seems to act primarily as a modifier of age at onset in individuals who are otherwise susceptible to AD. Almost twenty four years later, APOE4 remains the greatest genetic risk factor for Alzheimer's disease. There are several other genes researchers have linked with late onset AD including: SORL1, CLU, CR1, Phosphatidylinositol Binding Clathr in Assembly Protein (PICALM), and Triggering receptor expressed on myeloid cells 2 (TREM2). ${ }^{13}$ SORL1 is located on chromosome 11.

The CLU gene has been linked with $\mathrm{AD}$ due to the responsibility of the CLU gene is to regulate the clearance of amyloid-beta from the brain. Researchers suspect the imbalance in production and clearance of amyloid-beta is central to the development of Alzheimer's disease. Chronic inflammation in the brain has been linked to a deficiency in the CR 1 protein gene and inflammation is another possible factor 
for the development of Alzheimer's disease. It is important for proper neuron function in memory formation for smooth communication between neurons and the PICALM gene has been linked to the process of communication between neurons with brain nerve cells. TREM2 has recently been identified as a gene involved in the regulation of the brain's response to inflammation as well, and many variants within this gene have been associated with increased risks of Alzheimer's disease. Families with individuals with $\mathrm{AD}$ may inquire about genetic testing which has become more accessible through clinical laboratories and direct to consumer testing. The testing varies from early onset $A D$ vs late onset $\mathrm{AD}$ since there have been found different genetic differences. Genetic testing is available for early onset, autosomal dominant forms of Alzheimer's disease. Prenatal testing can also be performed if there have been genetic testing confirmed from prior family members with AD. Since multiple mutations in PSEN1, PSEN2, APP, and APOE can cause $\mathrm{AD}$, sequencing the entire coding regions of all genes is necessary to comprehensively assess the risk of $\mathrm{AD}$ and using this is not covered by commercial testing. Genetic counseling should be recommended prior to ordering tests and the genetic counselors have the responsibility to inform the patient and family of the options and implications of having a potentially inherited dominant mutation with almost complete penetrance. ${ }^{14}$ Some family members would rather not be informed of the possibility of an early onset $\mathrm{AD}$ diagnosis due to increased depression and inability to cope well with the results. ${ }^{15}$ It is ultimately up to the individual, if they choose to complete the genetic testing.

\section{Conclusion}

"The human brain contains billions of neurons that generate rhythmic and repetitive neural activity known as oscillations. These oscillations vary in time as repetitive measures about a central value, much like a pendulum or vibrating string, except that the wave generated in the human brain are electromagnetic. The difference in the electric potential between the two extreme oscillation points is defined as voltage and can be measured using electroencephalography (EEG). Traditionally used as an epilepsy diagnostic tool or adjunct test of brain death, EEGs can also be used to differentially diagnose between Alzheimer's disease and similar pathologies such as Lewy Body Disease (LBD). Since both anomalies affect the hippocampus it is especially difficult to discern between the two pathologies, except that LBD manifests a greater EEG wave deceleration". ${ }^{16}$ Alzheimer's disease continues as one of the most challenging disorders of the modern era and is considered a public health crisis. Alzheimer's disease effects on patients are devastating. The burden on a caregiver is dreadful, and the cost on the society is daunting. Understanding the genetic involvement of Alzheimer's disease may lead us to early detection, prevention, and ultimately definitive treatment or even a cure. In conclusion, the genetic factors of Alzheimer's disease remains poorly understood despite many advances within genetic testing. Further research initiatives are needed to revolutionize our understanding of the human mind and discover new ways to treat, prevent, and cure brain disorders such as Alzheimer's disease.

\section{Acknowledgements}

None.

\section{Conflict of interest}

Author declares that there is no conflict of interest.

\section{References}

1. Rudolph ET. The Genetics of Alzheimer's disease. Cold Spring Harbor Perspectives in Medicine. 2012;2(10):a006296.

2. Goate A, Chartier-Harlin MC, Mullan M, et al. Segregation of a missense mutation in the amyloid precursor protein gene with familial Alzheimer's disease. Nature. 1991;349(6311):704-706.

3. Renbaum P, Levy-Lahad E. Monogenic determinants of familial Alzheimer's disease: presenilin-2 mutations. Cell Mol Life Sci. 1998;54(9):910-919.

4. Rogaev EI, Sherrington R, Rogaeva EA, et al. Familial Alzheimer's disease in kindreds with missense mutations in a gene on chromosome 1 related to the Alzheimer's disease type 3 gene. Nature. 1995;376:775-778.

5. Selkoe DJ. Alzheimer's disease: genes, proteins, and therapy. Physiol Rev. 2001;81(2):741-766.

6. O’Brien RJ, Wong PC. Amyloid Precursor Protein Processing and Alzheimer's Disease. Annu Rev Neurosci. 2011;34:185-204

7. Brunkan AL, Goate AM. Presenilin function and gamma-secretase activity. J Neurochem. 2005;93(4):769-792.

8. Bettens K, Sleegers K, Van Broeckhoven C. Current status on Alzheimer's disease molecular genetics: from past, to present, to future. Hum Mol Genet. 2010;19(R1):R4-R11.

9. Sherrington R, Froelich S, Sorbi S, et al. Alzheimer's disease associated with mutations in presenilin 2 is rare and variably penetrant. Hum $\mathrm{Mol}$ Genet. 1996;5(7):985-988.

10. Tedde A, Nacmias B, Ciantelli M, et al. Identification of new presenilin gene mutations in early-onset familial Alzheimer's disease. Arch Neurol. 2003;60(11):1541-1544.

11. Wolozin B, Iwasaki K, Vito P, et al. Participation of presenilin 2 in apoptosis: enhanced basal activity conferred by an Alzheimer's mutation. Science. 1996;274(5293):1710-1713.

12. Blacker D, Tanzi D. The Genetics of Alzheimer's Disease: Current Status and Future Prospects. Arch Neurol. 1998;55(3):294-296.

13. Alzheimer's genes: Are you at risk? 2015.

14. Goldman JS, Hahn SE, Catania JW, et al. Genetic counseling and testing for Alzheimer's disease: joint practice guidelines of the American College of Medical Genetics and the National Society of Genetic Counselors. Genet Med. 2011;13(6):597-605.

15. Steinbart EJ, Smith CO, Poorkaj P, et al. Impact of DNA testing for earlyonset familial Alzheimer's disease and frontotemporal dementia. Arch Neurol. 2001;58(11):1828-1831.

16. Williamson J, Goldman J, Marder KS. Genetic Aspects of Alzheimer's Disease. Neurologist. 2009;15(2):80-86. 